\title{
Supporting Information: \\ How the Shape and Chemistry of Molecular Penetrants Control Responsive Hydrogel Permeability
}

\author{
Matej Kanduč, ${ }^{* \dagger}$ Won Kyu Kim, ${ }^{\ddagger}$ Rafael Roa, ${ }^{\top}$ and Joachim Dzubiella*,,$\|$ \\ $\dagger$ Jožef Stefan Institute, Jamova 39, SI-1000 Ljubljana, Slovenia \\ $\ddagger$ Korea Institute for Advanced Study, 85 Hoegiro, Seoul 02455, Republic of Korea \\ ФDepartamento de Física Aplicada I, Facultad de Ciencias, Universidad de Málaga, Campus de Teatinos s/n, E-29071 Málaga, \\ Spain \\ $\S$ Applied Theoretical Physics - Computational Physics, Physikalisches Institut, Albert-Ludwigs-Universität Freiburg, \\ Hermann-Herder Strasse 3, D-79104 Freiburg, Germany \\ ||Research Group for Simulations of Energy Materials, Helmholtz-Zentrum Berlin für Materialien und Energie, \\ Hahn-Meitner-Platz 1, D-14109 Berlin, Germany \\ E-mail: matej.kanduc@ijs.si; joachim.dzubiella@physik.uni-freiburg.de
}

\section{SIZE SCALING OF DIFFUSIVITY}

We fit the diffusivity data in the collapsed state $\left(D_{\mathrm{c}}\right)$ with three different exponential forms (for $n=1,2,3$ ):

$$
D_{\mathrm{c}}^{(n)}\left(a_{\mathrm{w}}\right)=D_{0} \exp \left(-\frac{a_{\mathrm{w}}{ }^{n}}{\lambda^{n}}\right)
$$

where $D_{0}$ and $\lambda$ are fitting parameters. The fits for the three different penetrant categories (spherical, planar $+\mathrm{Me}$, linear $+\mathrm{Me}$ ) are plotted in Figure S1A (for $n=1$; the same as Figure 2A in the main text), Figure S1B (for $n=2$ ), and Figure S1C (for $n=3$ ).

We compute the sum of squared residuals, which serves us to compare the quality of the fits:

$$
S^{(n)}=\sum_{i}\left[\ln D_{\mathrm{c}, i}-\ln D_{\mathrm{c}}^{(n)}\left(a_{\mathrm{w}, i}\right)\right]^{2}
$$

Table S1: Sum of squared residuals (eq S2) of the fits in Figure S1.

\begin{tabular}{llll} 
group & $S^{(1)}$ & $S^{(2)}$ & $S^{(3)}$ \\
\hline spherical & 0.35 & 3.27 & 7.17 \\
planar + Me & 0.17 & 0.47 & 0.99 \\
linear + Me & 0.31 & 0.70 & 1.17 \\
\hline \hline
\end{tabular}



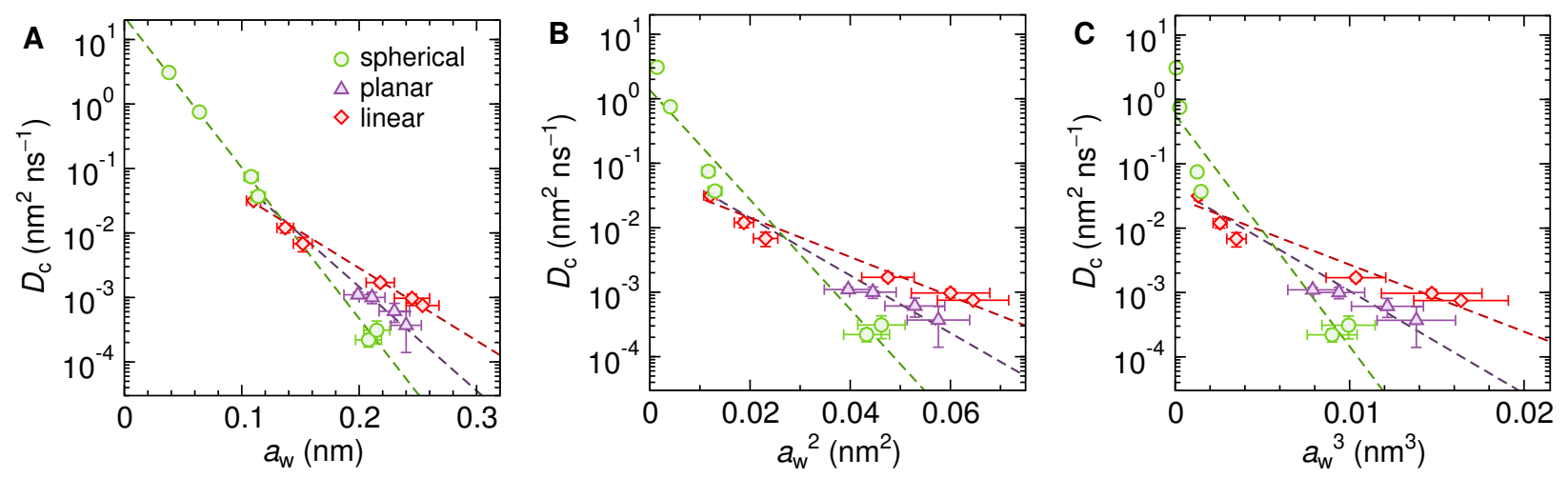

Figure S1: Diffusion coefficients of penetrants in the collapsed PNIPAM polymer (same data as in Figure 2A in the main text) plotted versus different powers of the penetrants' Stokes radii in water: (a) $a_{\mathrm{w}}$, (b) $a_{\mathrm{w}}{ }^{2}$, and (c) $a_{\mathrm{w}}{ }^{3}$. The dashed lines are fits of eq $\mathrm{S} 1$ to the three different categories of penetrants.

Visual inspection of the fits in Figure S1 as well as noticeably smaller values of $S^{(1)}$ than $S^{(2)}$ and $S^{(3)}$ in Table S1 clearly show that the linear form (i.e., $n=1$, which is eq 8 in the main text) provides by far the best description of the data among the three different popular possibilities suggested in the literature.

\section{GLASS TRANSITION TEMPERATURE}

We use two approaches to estimate the glass transition temperature, $T_{\mathrm{g}}$, of the collapsed PNIPAM model at $340 \mathrm{~K}$ (containing $20 \mathrm{wt} \%$ of water): (i) by cooling simulations and (ii) by monitoring water diffusivity at different temperatures.

Cooling simulations: In the first approach, we perform cooling simulations from an equilibrated structure at $400 \mathrm{~K}$ down to $100 \mathrm{~K}$ and monitor the density. The glass transition temperature is considered as the temperature at which the density vs. temperature slope undergoes a gradient change. The crossover is sensitive to the analysis-it depends on the cooling technique, statistical variation, cooling rate $(\Gamma)$, and the fitting range. ${ }^{1}$ To probe the latter two dependencies, we perform cooling simulations on three independent samples and at three different cooling rates, $\Gamma=5,20$, and $100 \mathrm{~K} \mathrm{~ns}^{-1}$. For fitting each branch, we use two different temperature intervals, $\Delta T=75 \mathrm{~K}$ and $100 \mathrm{~K}$ (i.e., $100-175 \mathrm{~K}$ and $100-200 \mathrm{~K}$ for the glassy branch and $325-400 \mathrm{~K}$ and $300-400 \mathrm{~K}$ for the rubbery branch).

An example of such a density-temperature plot is shown in Figure S2A, where $\Gamma=5 \mathrm{~K} \mathrm{~ns}^{-1}$. The resulting $T_{\mathrm{g}}$ averaged over the three independent samples are shown Figure S2B.

Water diffusivity: In the other approach, we perform longer simulations (1000 ns each) at different temperatures (ranging from 160 to $400 \mathrm{~K}$ ) of the system $(20 \mathrm{wt} \%$ water) and evaluate the diffusion coefficient of water. For even lower temperatures, the diffusion does not reach the normal diffusion regime within the simulation time, therefore the evaluation is not possible. Figure S3 shows an Arrhenius plot of the resulting diffusion coefficients, where a change in the slope is nicely visible. The crossover temperature obtained from linear fits is $T^{*}=275 \mathrm{~K}$. This agrees well with the $T_{\mathrm{g}}$ values obtained from the cooling method in Figure S2B.

From both analyses, we can thus confidently conclude that the glass transition temperature of our system is around $T_{\mathrm{g}}=275 \pm 10 \mathrm{~K}$, and the temperature to the glass transition temperature ratio is

$$
\frac{T}{T_{\mathrm{g}}} \approx 1.2
$$



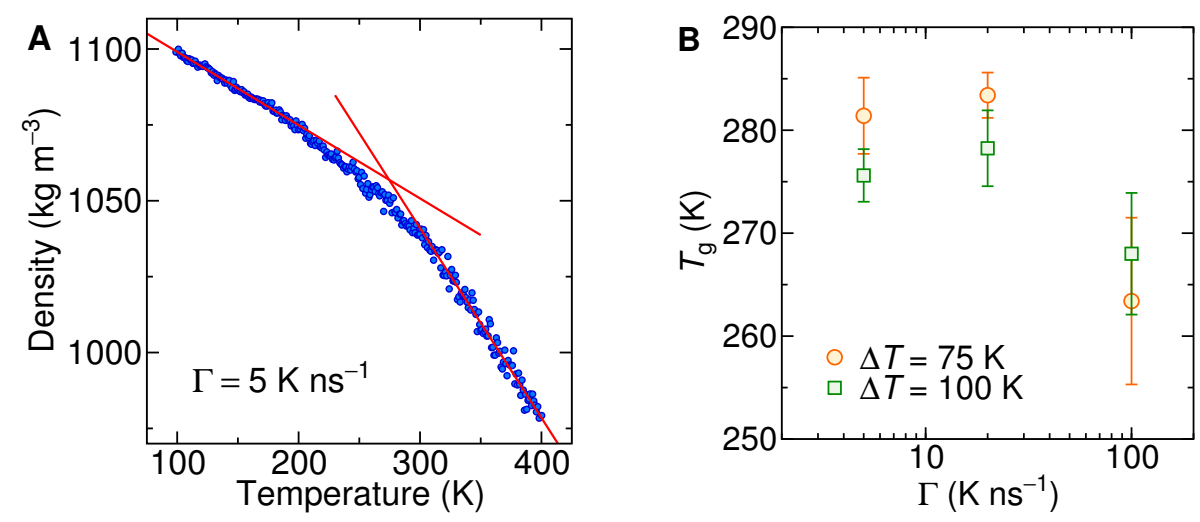

Figure S2: (A) Density-temperature curve at a cooling rate of $\Gamma=5 \mathrm{~K} \mathrm{~ns}^{-1}$. Red lines are linear fits to data on the outermost intervals of width $\Delta T=75 \mathrm{~K}$. (B) Evaluated glass transition temperature from the cooling method at different cooling rates and different fitting intervals.

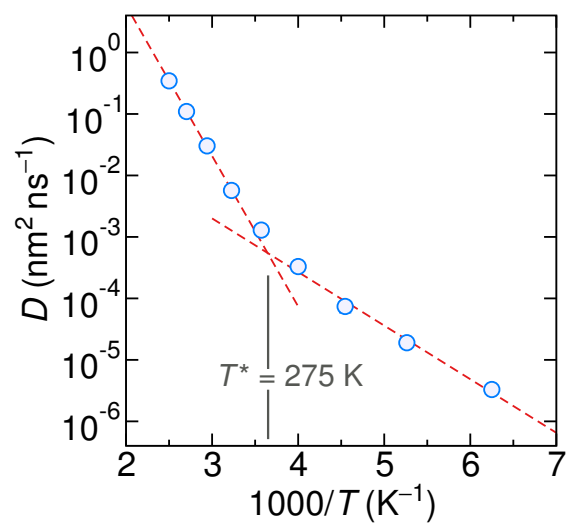

Figure S3: Arrhenius plot of the diffusion coefficient of water in the collapsed PNIPAM state. The water content in the simulations is fixed to $20 \mathrm{wt} \%$.

\section{CHAIN RELAXATION TIME}

For analyzing chain relaxation in the collapsed state at $340 \mathrm{~K}$, we use a self-intermediate scattering function ${ }^{2,3}$

$$
\left.F_{\mathrm{s}}(q, t)=\left\langle\cos \left\{\mathbf{q} \cdot\left[\mathbf{r}_{i}\left(t+t^{\prime}\right)\right]-\mathbf{r}_{i}\left(t^{\prime}\right)\right]\right\}\right\rangle_{i, t^{\prime}}
$$

which is based on the atomic displacements during the time interval $t$. The angle brackets $\langle\ldots\rangle_{i, t^{\prime}}$ denote the average over all chiral carbon atoms in the PNIPAM backbone and over time $t^{\prime}$. Our simulated systems are isotropic, therefore, the scattering function only depends on the absolute value of the scattering wave vector $q=|\mathbf{q}|$, determining the length scale at which dynamics is monitored.

Evaluated $F_{\mathrm{s}}(q, t)$ is plotted in Figure S4A as a function of time for different values of $q$. The time dependence can be described by a two-step relaxation ${ }^{3}$

$$
F_{\mathrm{s}}(q, t)=(1-A) \mathrm{e}^{-\left(t / \tau_{\mathrm{v}}\right)^{3 / 2}}+A \mathrm{e}^{-(t / \tau)^{\beta}}
$$

The first term reflects the fast dynamics modes of the polymer, and the second term describes the main relaxation 

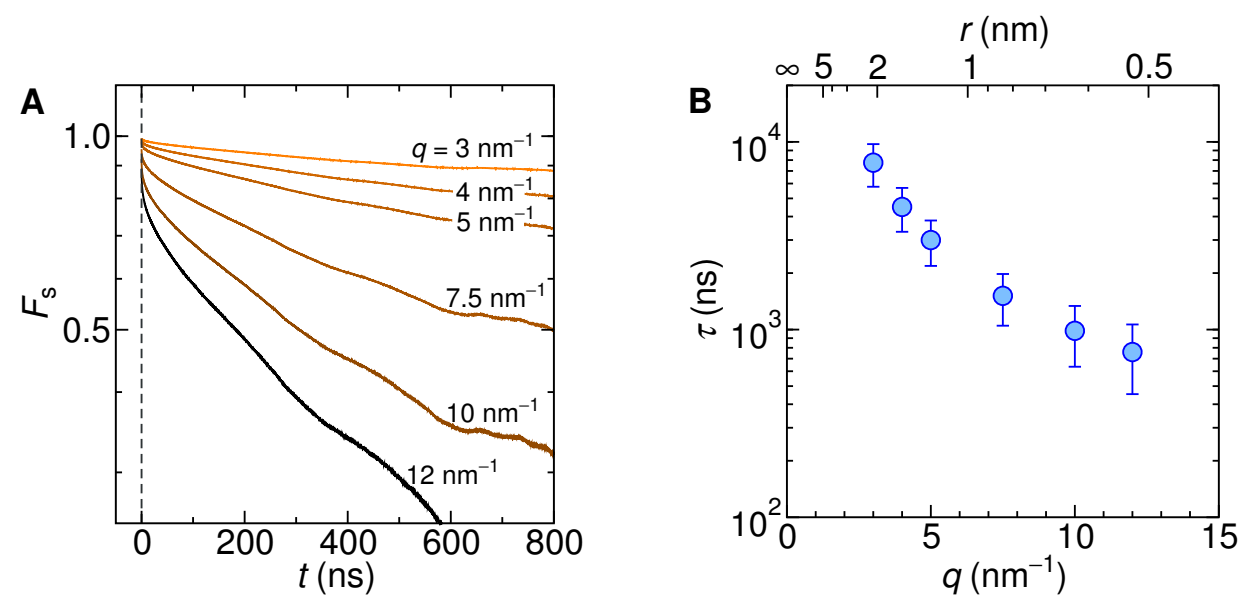

Figure S4: (A) Scattering function $F_{\mathrm{s}}(q, t)$ for the chiral carbon atoms of PNIPAM chains as a function of time at various wave vectors $q$. (B) Segmental relaxation times $\tau$ as a function of wave vector $q$ (bottom scale). The top scale shows the corresponding spatial dimension $r=2 \pi / q$.

mode of the polymer matrix. Here, $\beta$ stands for 'stretching exponent', empirically found $\beta<1$ for most cases. For simplicity, we will assume $\beta=1$ in our fits, which yields smaller values for the relaxation time $\tau$ than if assuming $\beta<1$. Since the fast relaxation dies out on the order much smaller than $100 \mathrm{~ns}$, we fit the second term of eq S5 to the data in Figure S4A in the range 100-500 ns. The fitted relaxation times $\tau$ as a function of wave vector are shown in Figure S4B.

A relevant length scale for diffusion processes is the distance between neighboring polymer chains. From the effective PNIPAM radius $R_{0}=0.5 \mathrm{~nm}$ and the fact that PNIPAM is a densely packed major component of the system ( $\left.80 \mathrm{wt} \%\right)$, the distance between chains is around $2 R_{0}=1 \mathrm{~nm}$, and the corresponding wave vector is $q=2 \pi / 2 R_{0} \approx 6 \mathrm{~nm}^{-1}$. The chain relaxation time at this length scale is around $2 \times 10^{3} \mathrm{~ns}$ (see Figure S4B).

\section{COMPARISON WITH THE SELF-CONSISTENT COOPERATIVE HOPPING THEORY}

We compare the scaling of diffusion resulting from our simulations with predictions of the self-consistent cooperative hopping theory (SCCHT) by Zhang and Schweizer. ${ }^{4}$ The SCCHT is namely one of a few theories that predict a near exponential decrease of penetrant diffusivity with its size. The theory is based on the activated diffusion of spherical penetrants in dense melts and glasses of matrix spheres. The predictions for diffusion can be approximately described with the equation

$$
D_{\text {hop }} / D_{0}=\exp \left(-\xi \frac{d}{\sigma}\right)
$$

where $\sigma$ is the diameter of the matrix sphere, $d$ the diameter of the penetrant, and the dimensionless parameter $\xi$ depends on the matrix volume fraction $\phi_{\mathrm{m}}$. By fitting eq S6 to the predictions for various $\phi_{\mathrm{m}}$ in Figure 3 in ref 4 on the interval of $d / \sigma$ from 0.3 to 0.6 , we obtain coefficients $\xi$ shown in Table S2. As it turns out, the penetrant-matrix interaction does not significantly change the slope (i.e., $\xi$ ) but instead modifies only the prefactor $D_{0}$ in eq S6 (see Figures 8 and 9 in ref 4 ).

To quantitatively compare the predictions of the SCCHT with our simulations, we have to map our molecular model to an effective mixture of spheres. To do that, we interpret the matrix sphere radius $\sigma$ in the SCCHT as the Kuhn length of the polymer chains in our model, $\sigma=L_{\mathrm{K}}$, as proposed in ref 4 . With that, we can rewrite eq S6 directly to eq 8 in the main text by matching the exponents, $\xi(d / \sigma)=(d / 2) / \lambda$, which provides the parameter $\lambda$ in our notation as

$$
\lambda=\frac{L_{\mathrm{K}}}{2 \xi}
$$


Table S2: Parameter $\xi$ in eq S6 evaluated from ref 4 at different matrix volume fractions $\phi_{\mathrm{m}}$. Parameter $\lambda$ follows from eq S7.

\begin{tabular}{lll}
$\phi_{\mathrm{m}}$ & $\xi$ & $\lambda(\mathrm{nm})$ \\
\hline 0.63 & 55.5 & 0.010 \\
0.611 & 39.7 & 0.014 \\
0.6 & 33.5 & 0.017 \\
0.59 & 28.3 & 0.020 \\
0.57 & 20.9 & 0.027 \\
\hline \hline
\end{tabular}

This allows us to evaluate the effective $\lambda$ from the SCCHT and compare it to our simulations.

Before doing so, we have to extract the Kuhn length of PNIPAM chains from our simulations, which is defined as

$$
L_{\mathrm{K}}=\frac{\left\langle r_{\mathrm{ee}}^{2}\right\rangle}{L_{\mathrm{c}}}
$$

where $r_{\mathrm{ee}}^{2}$ is the mean square distance between the ends of the chain (in our case defined by the chiral carbon atoms in the PNIPAM backbone) and $L_{\mathrm{c}}=5.0 \mathrm{~nm}$ is the contour length of the backbone of the chain. Averaging $r_{\mathrm{ee}}^{2}$ over time and over all chains in the system (denoted by angle brackets), gives us $L_{\mathrm{K}}=1.14 \mathrm{~nm}$.

The calculated values for $\lambda$ from the SCCHT, obtained from eq S7, is given in Table S2. In the end, we want to find a reasonable estimate for the matrix volume fraction $\phi_{\mathrm{m}}$ of the hard-sphere system that corresponds best to our molecular model. The volume fraction of the close-packed spheres equals $\phi_{\max } \approx 0.74$, which can be considered as a completely dehydrated, densely packed polymer system. Our system at $340 \mathrm{~K}$ contains 20 wt $\%$ of water and 80 $\mathrm{wt} \%$ of the polymer, thus a reasonable mapping to the hard-sphere model is $\phi_{\mathrm{m}}=\phi_{\max } \times 0.80 \approx 0.59$. As seen from Table S2, at this particular volume fraction the value for $\lambda$ from the SCCHT is $0.020 \mathrm{~nm}$, whereas our simulations give $\lambda=0.0186 \mathrm{~nm}$ for spherical penetrants (Table 1 in the main text), which is very good agreement.

\section{COMPRESSIBILITY OF THE COLLAPSED PNIPAM STATE}

In order to evaluate the compressibility of the collapsed PNIPAM state, we perform additional simulations (three independent samples) of the PNIPAM at $340 \mathrm{~K}$ at the pressure of 1000 bar for $70 \mathrm{~ns}$. We discard the first $10 \mathrm{~ns}$ for relaxation reasons and evaluate the volume, shown in Table S3.

Table S3: Mean simulation box volume at two different pressures.

\begin{tabular}{rl}
$p$ (bar) & $V\left(\mathrm{~nm}^{3}\right)$ \\
\hline 1 & $214.5(1)$ \\
$10^{3}$ & $207.4(2)$ \\
\hline \hline
\end{tabular}

The isothermal compressibility is then computed as

$$
\chi_{\mathrm{p}}=-\frac{1}{V} \frac{\Delta V}{\Delta p}
$$

which gives $\chi_{\mathrm{p}}=3.3(1) \times 10^{-5} \mathrm{bar}^{-1}$. This is smaller than the compressibility of bulk water at $340 \mathrm{~K}, \chi_{\mathrm{w}}=$ $4.65(1) \times 10^{-5}$ bar $^{-1}$. 


\section{SIZE SCALING OF TRANSFER FREE ENERGY}

We fit the transfer free energy data from water into the collapsed PNIPAM state $(\Delta G)$ with two different forms

$$
\begin{aligned}
\Delta G^{(A)} & =\Delta G_{0}+4 \pi \gamma_{0}\left(a_{\mathrm{AS}}\right)^{2} \\
\Delta G^{(V)} & =\Delta \tilde{G}_{0}+\frac{4}{3} \pi \rho_{0}\left(a_{\mathrm{AS}}\right)^{3}
\end{aligned}
$$

which are shown in Figure S5.
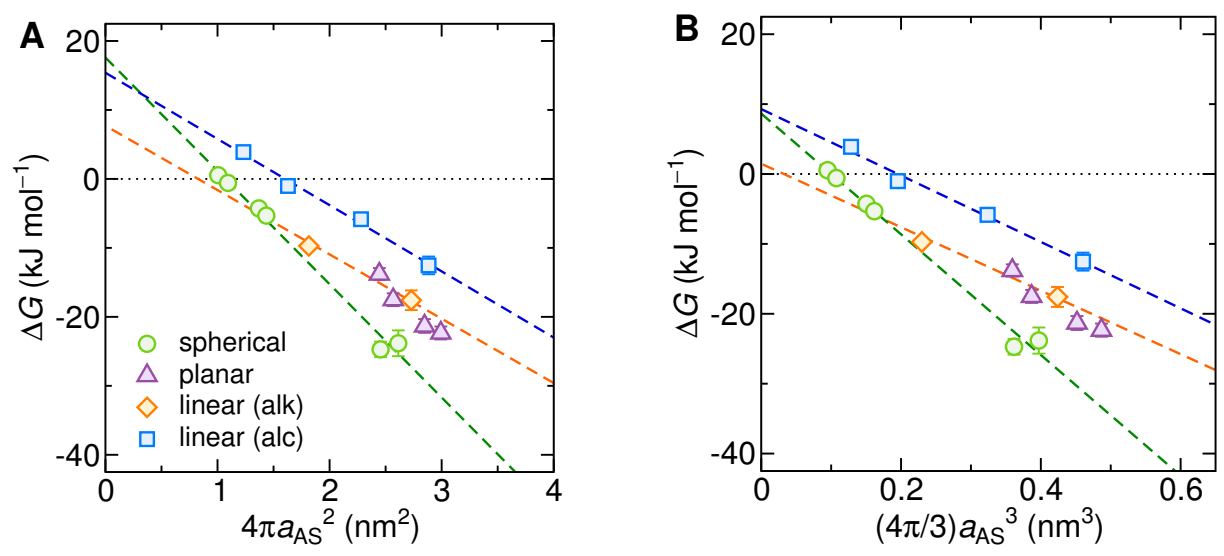

Figure S5: Transfer free energies of penetrants from water into the collapsed PNIPAM (same data as in Figure 2B in the main text) plotted versus penetrant (A) surface area and (B) volume. The dashed lines are fits of eqs S10 and S11 to the different categories of penetrants in panels A and B, respectively.

We asses the quality of the fits by computing the sum of squared residuals,

$$
\begin{aligned}
S^{(A)} & =\sum_{i}\left[\Delta G_{i}-\Delta G^{(A)}\left(a_{\mathrm{AS}, i}\right)\right]^{2} \\
S^{(V)} & =\sum_{i}\left[\Delta G_{i}-\Delta G^{(V)}\left(a_{\mathrm{AS}, i}\right)\right]^{2}
\end{aligned}
$$

Table S4: Sum of squared residuals (eqs S12 and S13, in the units of $\mathrm{kJ}^{2} \mathrm{~mol}^{-2}$ ) of the fits in Figure S5.

\begin{tabular}{lcc} 
group & $S^{(A)}$ & $S^{(V)}$ \\
\hline spherical & 7.33 & 7.88 \\
linear (alkanes) + $\mathrm{Me}$ & 1.20 & 1.61 \\
linear (alcohols) $+\mathrm{H}_{2} \mathrm{O}$ & 0.41 & 0.91 \\
\hline \hline
\end{tabular}

Comparing the fits in Figure S5 by visual inspection, it is difficult to discern the difference in the fitting quality. However, the squared residuals in Table S4 demonstrate better fits with surface area scaling (eq S10) for all three groups. 


\section{References}

(1) McKechnie, D.; Cree, J.; Wadkin-Snaith, D.; Johnston, K. Glass Transition Temperature of a Polymer Thin Film: Statistical and Fitting Uncertainties. Polymer 2020, 122433.

(2) Bormuth, A.; Henritzi, P.; Vogel, M. Chain-Length Dependence of the Segmental Relaxation in Polymer Melts: Molecular Dynamics Simulation Studies on Poly (Propylene Oxide). Macromolecules 2010, 43, 8985-8992.

(3) Starr, F. W.; Douglas, J. F.; Meng, D.; Kumar, S. K. Bound Layers "Cloak" Nanoparticles in Strongly Interacting Polymer Nanocomposites. ACS nano 2016, 10, 10960-10965.

(4) Zhang, R.; Schweizer, K. S. Correlated Matrix-Fluctuation-Mediated Activated Transport of Dilute Penetrants in Glass-Forming Liquids and Suspensions. J. Chem. Phys. 2017, 146, 194906. 